\title{
Anorexie mentale et trouble du comportement alimentaire selon une perspective phénoménologique : version francophone du questionnaire IDentity and EAting disorders $(\text { IDEA) })^{\text {is }}$
}

\section{Anorexia nervosa and eating disorders from a phenomenological perspective: A French version of the IDentity and EAting disorders (IDEA) questionnaire}

Jérôme Englebert (Docteur en psychologie) ${ }^{\mathrm{a}, * \mathrm{~b}}$, Eugénie Minguet (Psychologue) a, Adam Helinski (Psychologue) ${ }^{\mathrm{a}}$, Françoise Dominé (Psychiatre) ${ }^{\mathrm{c}}$, Sandra Gebhard (Psychiatre) ${ }^{\mathrm{d}}$, Catherine Zdrojewski (Infirmière) ${ }^{\mathrm{b}}$, Giovanni Castellini (Professeur) ${ }^{\mathrm{e}}$, Valdo Ricca (Professeur) ${ }^{\mathrm{e}}$, Milena Mancini (Psychologue) ${ }^{\mathrm{f}}$, Friedrich Stiefel (Professeur) ${ }^{\mathrm{b}}$, Giovanni Stanghellini (Professeur) ${ }^{\mathrm{f}}$, Adélaïde Blavier (Professeur) ${ }^{\mathrm{a}}$

\footnotetext{
a Département de psychologie, centre d'expertise en psychotraumatisme et psychologie légale, université de Liège (Belgique) Quartier Agora, place des Orateurs 1 (Bât B33), 4000 Liège, Belgique

b Service de psychiatrie de liaison, centre hospitalier universitaire Vaudois, université de Lausanne (Suisse), rue du Bugnon 46, 1005 Lausanne, Suisse
}

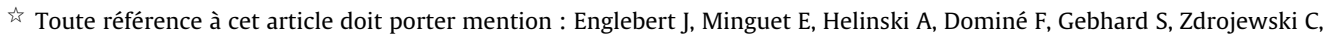
Castellini G, Ricca V, Mancini M, Stiefel F, Stanghellini G, Blavier A. Anorexie mentale et trouble du comportement alimentaire selon une perspective phénoménologique : Version francophone du questionnaire IDentity and EAting disorders (IDEA). Evol psychiatr 2019; 84 (4): pages (pour la version papeir) ou URL [date de consultation] (pour la version électronique).

* Auteur correspondant.

Adresse e-mail : jerome.englebert@uliege.be (J. Englebert).
} 
' Département de pédiatrie, CHR La Citadelle, Liège (Belgique), boulevard du Douzième-de-Ligne 1, 4000

Liège, Belgique

d Centre vaudois anorexie boulimie, centre hospitalier universitaire vaudois, université de Lausanne

(Suisse), rue du Tunnel 1, 1005 Lausanne, Suisse

e Dipartimento di scienze della Salute, università di Firenze (Italie), Largo Brambilla, 3, 50134 Firenze, Italie

f Department of humanitistic, psychological and territorial sciences, Università "G. d'Annunzio" - Chieti

(Italie), Via Pescara, 66100 Chieti, Italie

\section{N F O A A R T I C L E}

Historique de l'article :

Reçu le 5 mars 2019

\section{Mots clés :}

Anorexie mentale

Phénoménologie

Corps-pour-autrui

Incarnation

Identité

Questionnaire

Identity and Eating Disorders (IDEA)

\section{R É S U M É}

Objectif. - Cet article présente la version francophone du questionnaire d'inspiration phénoménologique Identity and Eating Disorders (IDEA) (Stanghellini et al., 2012). La littérature d'inspiration phénoménologique met en évidence que les personnes souffrant d'anorexie mentale présentent des perturbations au niveau de l'incarnation et de l'intersubjectivité. Cette recherche évalue les expériences corporelles subjectives de participantes anorexiques grâce au concept du corps-pour-autrui proposé par J.-P. Sartre (1943).

Hypothèse. - Les patientes anorexiques se définissent davantage à travers le regard des autres et tendent à moins ressentir leur corps de manière sensorielle.

Méthode. - Soixante-sept patientes anorexiques et 246 participantes contrôles ont répondu à la version francophone, traduite par nos soins, du questionnaire IDentity and EAting disorders. Des tests non paramétriques de Mann-Whitney pour échantillons indépendants ont été effectués.

Conclusion. - Les résultats statistiques confirment l'hypothèse de recherche : les patientes anorexiques obtiennent des scores au questionnaire significativement supérieurs à ceux des participantes contrôles. Ces résultats confirment des difficultés au niveau de l'incarnation et de l'intersubjectivité chez les sujets anorexiques et annoncent des perspectives de recherche importantes.

(c) 2019 Elsevier Masson SAS. Tous droits réservés.

\section{A B S T R A C T}

Objective. - This research set out to test the French version of the IDentity and EAting disorders questionnaire (IDEA) (Stanghellini et al., 2012). From a phenomenological perspective, anorexic patients seem to experience difficulties regarding the subjective feeling of their own body (embodiment) and their personal identity, which leads to an impairment of inter-subjectivity in social interactions. Following these findings, this study investigated the "body-for-others" concept (Sartre, 1943) in a sample of anorexic female patients. These patients tended to define themselves mostly through the eyes of others instead of from their own perspective: their Leib (i.e. the real body) was hypothesized to be more vulnerable than their Körper (i.e. the body object).

Hypothesis. - The main hypothesis was that anorexic patients would define themselves mostly through the eyes of others and would tend to experience fewer bodily sensations than control participants. Significantly higher scores for these dimensions in the clinical group compared to the control group were expected. 
Method. - This study included 67 anorexic female patients diagnosed on the Diagnostic and Statistical Manual IV criteria, and 246 female controls. All the participants were French speakers from Belgium, France and Switzerland. Participants were asked to complete two self-report questionnaires: The Eating Disorder Inventory 2 (EDI-2) (French version) evaluating eating disorders and the IDEA (French version translated and adapted by the authors) to explore the "body-for-others" concept. Mann-Whitney non-parametric tests were used to compare the results for the clinical and the control groups.

Conclusion. - The outcomes confirmed our hypothesis: the anorexic female patients had significantly higher scores compared to the control group on both of the questionnaires: the EDI- 2 and the IDEA. The anorexic patients seemed to have difficulties in the way they experienced their own body, their personal identity and their perceptions of the gaze of others. The IDEA is a tool that has been elaborated for the assessment of these issues. It enriches knowledge relating to the body-self relationship and the first-person perspective, which is essential for a phenomenological approach. However, it still requires further research to be validated and used in clinical practice.

(C) 2019 Elsevier Masson SAS. All rights reserved.

\section{Introduction}

L'anorexie mentale est une pathologie psychiatrique appartenant à la catégorie des troubles du comportement alimentaire au même titre que la boulimie, l'hyperphagie boulimique et les troubles du comportement alimentaire non spécifiés selon les DSM-IV et DSM-5 [1-3]. L'anorexie mentale affecte surtout les femmes avec un ratio de 9:1 à 10:1 [4] et se manifeste généralement entre 12 et 18 ans [5]. Il est important de rappeler que ce syndrome figure parmi les troubles psychiatriques les plus mortels avec un taux de mortalité à 10 ans de $5 \%$ [2]. La moitié des décès est attribuée à l'état de dénutrition et l'autre moitié au suicide [6]. En ce qui concerne l'évolution des patientes anorexiques, on considère que $50 \%$ des cas guérissent, 30 \% présentent des rechutes au cours de leur vie et $20 \%$ se chronicisent [7].

L'approche phénoménologique en psychopathologie est une approche qualitative qui consiste en une prise en considération des expériences vécues et relatées par le sujet. Selon cette perspective, le chercheur/clinicien s'intéresse à comprendre la manière d'être-au-monde spécifique de chaque individu et ses états de subjectivité [8,9]. Les propositions de ce courant reposent, dès lors, sur une perspective en première personne contrastant avec l'approche en troisième personne qui est une perspective extérieure à l'individu, généralement adoptée par le milieu médical et les sciences cognitives [8].

Selon la perspective phénoménologique, la problématique anorexique (y compris la défaillance dans la régulation du poids et l'amincissement extrême) s'inscrirait dans une perturbation profonde de l'identité et de l'incarnation, autrement dit, de la manière dont les personnes concernées " habitent " et ressentent leur corps. Les comportements alimentaires pathologiques seraient, par conséquent, des symptômes secondaires-pourtant particulièrement visibles-de ces perturbations plus fondamentales [10,11]. L'approche phénoménologique des Troubles du Comportement Alimentaire (TCA) porte son attention sur les perceptions subjectives du corps, de l'identité, de l'espace, du temps, du contrôle et de la perte du contrôle $[10,12-23]$.

Des travaux récents $[15,19,20]$ ont proposé un outil phénoménologique permettant d'approfondir l'hypothèse concernant la place du corps-pour-autrui dans le vécu des personnes souffrant de TCA : l'IDEA (IDentity and EAting disorders) qui est un questionnaire auto-rapporté en première personne focalisé sur l'expérience subjective des participants. Il s'agit du premier questionnaire psychopathologique fondé sur le concept du corps-pour-autrui, issu de la philosophie de Jean-Paul Sartre (1942) 
[24]. Les résultats de ces études indiquent que le questionnaire IDEA se révèle prédictif par rapport au développement d'un TCA dans un futur proche en fonction des évènements de vie [15,19,20]. L'ajout de ce questionnaire enrichit la connaissance concernant le rapport au corps des patientes et plus particulièrement la dimension relationnelle de celui-ci. L'objectif du présent article est de proposer une validation francophone du questionnaire IDEA, administré à une population de femmes anorexiques $(\mathrm{n}=67)$. Soulignons que l'étude source se répartissait de la sorte : 50 sujets anorexiques, 48 boulimiques et 49 souffrant de binge eating $(n=147)$.

\section{Hypothèse psychopathologique}

La tradition phénoménologique envisage deux corps : le Körper, autrement dit le corps physique, et le Leib, l'expérience subjective corporelle. Sartre propose un troisième concept se rapportant au corps : le corps-pour-autrui ([24], p. 379). Celui-ci fait référence à notre capacité d'appréhender notre corps d'un point de vue extérieur à nous-même, plus particulièrement du point de vue de l'autre lorsqu'il nous regarde. En réalisant que notre corps peut être observé par l'autre, nous comprenons que notre corps devient un objet pour celui-ci. L'expérience corporelle n'est dès lors plus uniquement une expérience en première personne vécue de l'intérieur, mais également une perspective en troisième personne où le corps devient l'objet du regard d'autrui sur lequel l'individu n'a pas de prise.

Plusieurs études $[16,17,19]$ suggèrent que les patients souffrant de TCA expérimentent leur corps en surinvestissant précisément cette manière de le vivre, comme s'ils étaient constamment regardés, observés et jugés. Par ce processus, leurs expériences subjectives comme les sensations et leur sentiment d'agentivité sur le monde leur échappent en partie. De façon plus spécifique, c'est surtout le trouble anorexique qui a été étudié selon ces modélisations phénoménologiques [14-17,23] et l'on y décrit un déséquilibre entre le corps-sujet-percevant et le corps-objet-perçu en faveur de ce dernier $[9,10,12,13]$.

Le propos de cette recherche concerne l'étude des difficultés expérimentées par la personne anorexique par rapport aux phénomènes d'intersubjectivité. Nous reprenons donc l'hypothèse de l'étude source de l'IDEA soutenant que les patientes anorexiques tendent à moins ressentir leur corps de manière sensorielle et accordent au regard qu'autrui pose sur elles (et pose sur leur corps) une importance excessive comparativement à des personnes ne présentant pas d'anorexie.

\section{Populations}

Cette recherche comprend deux groupes. Le groupe clinique, composé de participantes souffrant d'anorexie mentale, et le groupe contrôle, constitué de participantes ne présentant pas de TCA. Toutes sont âgées d'au moins 16 ans et francophones. Celles-ci, ainsi que les parents pour les sujets mineurs, ont signé un consentement éclairé et la recherche a été avalisée par différents comités d'éthique (Faculté de Psychologie, Logopédie et Sciences de l'Éducation de l'Université de Liège et Faculté de Médecine de l'Université de Lausanne). L'échantillon ne comporte pas de sujet masculin.

\subsection{Population anorexique}

Le groupe clinique est constitué de 67 patientes anorexiques âgées de 16 à 53 ans (voir Tableau 1). Sur les 67 patientes composant l'échantillon clinique, 13 patientes anorexiques sont belges,

Tableau 1

Moyennes et écarts-types de l'IMC et de l'âge des groupes contrôle et anorexique.

\begin{tabular}{llllll}
\hline & \multicolumn{2}{l}{ Groupe contrôle $(n=246)$} & & & Groupe anorexique $(n=67)$ \\
\cline { 2 - 3 } & Moyenne & Écart-type & & Moyenne & Écart-type \\
\hline IMC & 20,88 & 2,00 & 16,04 & 1,20 \\
Âge & 23,26 & 6,19 & 24,93 & 8,94 \\
\hline
\end{tabular}

$n=$ nombre de participantes ; IMC = Indice de Masse Corporelle (le poids divisé par la taille au carré). 
28 françaises et 26 suisses. Toutes les patientes recrutées répondent aux critères d'anorexie mentale du DSM-5. Cependant, nous n'avons pas tenu compte du type d'anorexie (restrictif ou avec ou sans crises de boulimie) au vu de la labilité du diagnostic [25] (Tableau 1).

\subsection{Population contrôle}

Le groupe contrôle est composé de 246 participantes âgées de 16 à 58 ans (voir Tableau 1) ne présentant pas et n'ayant jamais présenté de TCA. Pour maximiser les chances d'obtenir un échantillon représentatif, les participantes présentant un IMC inférieur à 17,5 ou supérieur à 25 n'ont pas été retenues, ainsi que celles ayant présenté des fluctuations pondérales importantes au cours de leur vie (entre 10 et $20 \mathrm{~kg}$ ). Sur les 246 participantes, 136 sont belges, 81 ont répondu à l'annonce mise sur les réseaux sociaux ( 78 sont françaises et 3 sont suisses) et 29 proviennent d'une haute école de Lausanne.

Comme l'indique le Tableau 1, l'IMC moyen du groupe de participantes anorexiques est significativement inférieur à celui de la population contrôle $(\mathrm{U}=47$ et $p<0,00000001)$. Alors que l'IMC moyen du groupe contrôle correspond à la catégorie normale (la catégorie de l'IMC moyen selon la World Health Organization [26] varie entre 18,5 et 24,9), celui du groupe anorexique est nettement en-dessous. Par rapport à l'âge, il n'y a pas de différence significative entre les participantes anorexiques et les participantes contrôles ( $\mathrm{U}=7529$ et $p=0,99$ ) (le test non paramétrique de Mann-Whitney pour échantillons indépendants a été effectué).

\section{Questionnaires auto-rapportés}

\subsection{Eating Disorder Inventory-2 (EDI-2)}

Pour évaluer et contrôler la présence d'un TCA, la version française du Eating Disorder Inventory 2 (EDI-2, [27]) a été choisie. Ce questionnaire comprend 91 items dont 65 items directs et 26 indirects. Pour chaque item, les participantes doivent indiquer leur degré d'accord selon une échelle de Likert à 6 modalités de réponses correspondant à : toujours (3), en général (2), souvent ( 1 ), quelquefois ( 0 ), rarement $(0)$, jamais $(0)$ pour les items directs et à : toujours ( 0 ), en général $(0)$, souvent $(0)$, quelquefois (1), rarement (2), jamais (3) pour les items indirects. L'EDI-2 comprend 11 facteurs : la recherche de la minceur, la boulimie, l'insatisfaction corporelle, le sentiment d'inefficacité, le perfectionnisme, la méfiance interpersonnelle, la conscience intéroceptive, la peur de la maturité, l'ascétisme, le contrôle des pulsions et l'insécurité sociale.

\subsection{IDentity and EAting disorders (IDEA)}

Pour évaluer le concept du corps-pour-autrui, la version francophone traduite par nos soins (voir Annexe 1), du questionnaire IDEA: IDentity and EAating disorders [19] a été utilisée. Ce questionnaire est constitué de 27 items pour lesquels les participantes doivent indiquer leur degré d'accord selon une échelle de Likert à 5 modalités de réponses : pas du tout (0), légèrement (1), moyennement (2), beaucoup (3), fortement (4). Les items sont regroupés en quatre facteurs : Feeling oneself through the gaze of the other and oneself through the evaluation of the other que nous traduisons par : " se sentir soi-même à travers le regard et l'évaluation des autres " (Facteur GEO), Feeling oneself through objective measures : "se sentir soi-même à travers des mesures objectives " (Facteur OM), Feeling extraneous from one's own body: " se sentir étranger à son corps " (Facteur EB) et Feeling oneself through starvation : " se sentir soi-même à travers la privation " (Facteur $\mathrm{S}$ ).

En ce qui concerne la traduction du questionnaire, une première version a été réalisée par E. Minguet et J. Englebert. Elle a été revue par l'ensemble des contributeurs de l'article, en particulier les auteurs de l'étude source à l'origine du questionnaire (sous la responsabilité de G. Stanghellini). Après accord pour une première version, celle-ci a été testée, d'abord sur cinq sujets « contrôles », ensuite sur cinq sujets anorexiques qui n'ont pas étés inclus dans les résultats.

Pour chaque facteur, la moyenne des items est calculée. Puisque le questionnaire IDEA mesure différentes facettes du corps-pour-autrui, un score total, équivalent à la moyenne des quatre facteurs, est calculé. 


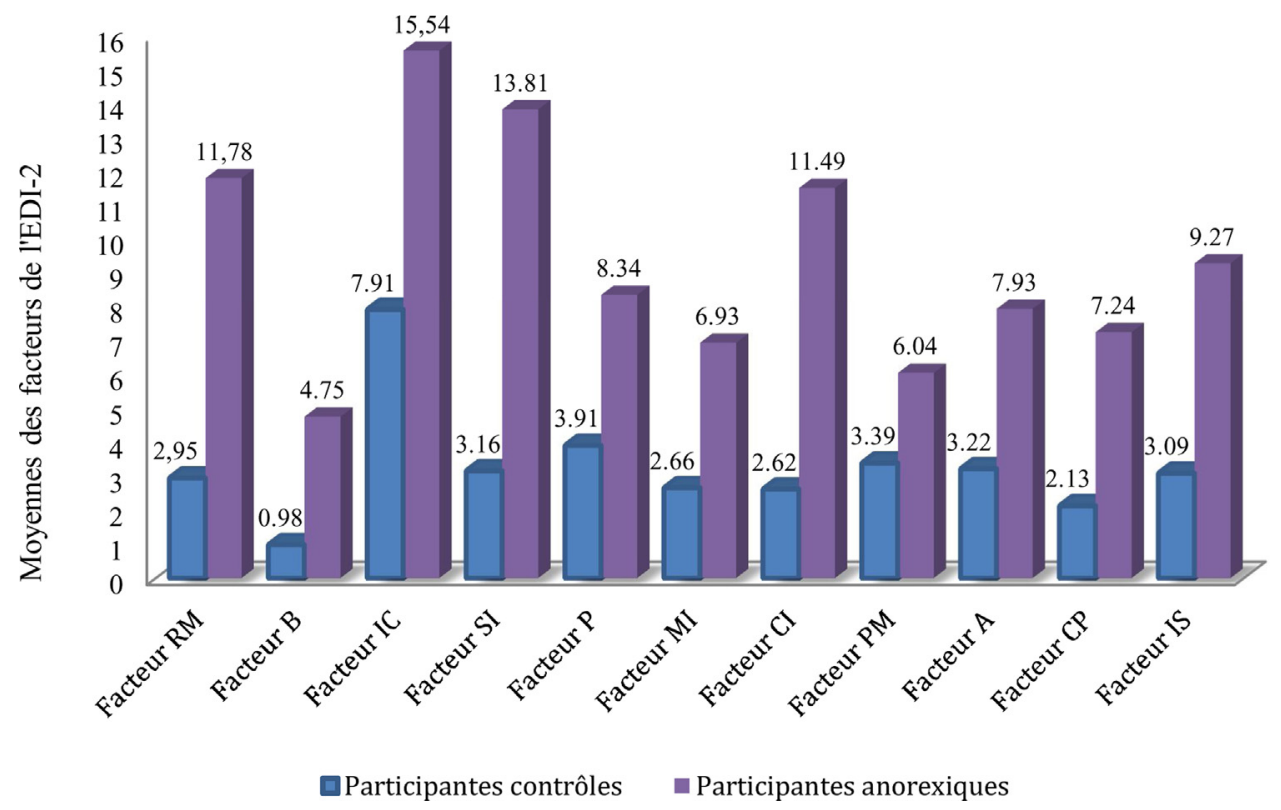

Fig. 1. Moyennes des facteurs de l'EDI-2 en fonction des groupes de participantes. Facteur RM = recherche de la minceur ; Facteur $\mathrm{B}$ = boulimie ; Facteur IC = insatisfaction corporelle ; Facteur $\mathrm{SI}=$ sentiment d'inefficacité ; Facteur $\mathrm{P}=$ perfectionnisme ; Facteur $\mathrm{MI}=$ méfiance interpersonnelle ; Facteur $\mathrm{CI}=$ conscience intéroceptive ; Facteur $\mathrm{PM}=$ peur de la maturité ; Facteur $\mathrm{A}=$ ascétisme ; Facteur $\mathrm{CP}=$ contrôle des pulsions ; Facteur IS = insécurité sociale.

\section{Analyses statistiques}

Les analyses statistiques ont été réalisées avec le logiciel STATISTICA. Concernant la normalité de la distribution des variables, les tests de normalité de Shapiro-Wilk effectués sur celle-ci indiquent un rejet de l'hypothèse nulle. Autrement dit, la distribution des variables étudiées ne respecte pas la normalité. Des tests statistiques non-paramétriques ont donc été utilisés. Pour comparer les résultats entre le groupe clinique anorexique et le groupe contrôle, des tests de Mann-Whitney (avec une probabilité de dépassement fixée à 0,05 ) ont été effectués par rapport aux moyennes des facteurs aux différents questionnaires.

De plus, pour établir les liens entre les différents questionnaires dans le groupe de participantes anorexiques, nous avons testé les corrélations entre les facteurs de l'IDEA et les facteurs de l'EDI-2. Des corrélations de Spearman, qui équivalent aux corrélations de Pearson en version non-paramétrique, ont été utilisées.

\section{Résultats}

\subsection{Les résultats du questionnaire EDI-2}

Le questionnaire EDI-2 permet de distinguer certains facteurs caractéristiques des TCA. Cette ligne de base nous permet ainsi de vérifier si le groupe de patientes anorexiques est bien concerné par ces traits. Inversement, des résultats plus faibles aux facteurs sont attendus pour le groupe contrôle. Comme prévu, les groupes contrôle et anorexique obtiennent des résultats statistiquement différents aux onze facteurs évalués (voir Fig. 1). Le test statistique de Mann-Whitney pour échantillons indépendants comparant les moyennes des groupes contrôle et anorexique aux facteurs du questionnaire EDI-2 met en évidence des valeurs significatives ( $p$ compris entre $<0,001$ et $<0,000001$ ). Nous pouvons interpréter ces résultats comme un gage de représentativité des échantillons respectifs. 


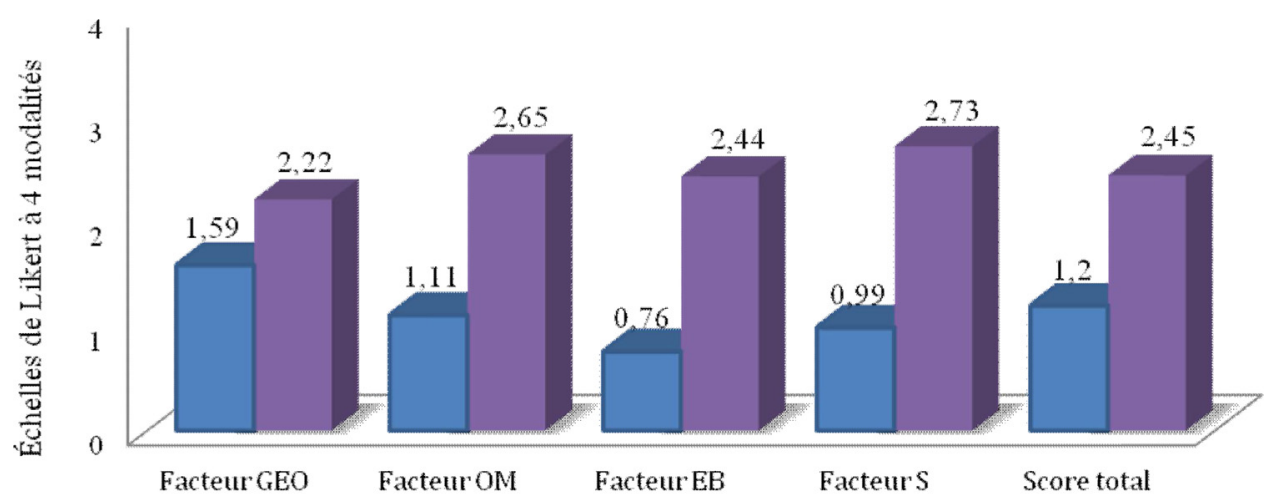

aparticipantes contrôles = Participantes anorexiques

Fig. 2. Moyennes des facteurs de l'IDEA en fonction des groupes de participantes. Facteur GEO=se sentir soi-même à travers le regard et l'évaluation des autres ; Facteur $\mathrm{OM}=$ se sentir soi-même à travers des mesures objectives ; Facteur $\mathrm{EB}=$ se sentir étranger à son corps ; Facteur $\mathrm{S}=$ se sentir soi-même à travers la privation alimentaire ; Score total=score moyen au questionnaire.

En comparant les moyennes du groupe contrôle avec celles de la norme contrôle de la version anglaise de Garner [27], nous constatons que nos moyennes sont similaires excepté pour trois facteurs (recherche de la minceur, insatisfaction corporelle et perfectionnisme) où les moyennes de nos participantes contrôles sont moins élevées, sans toutefois dépasser un écart-type associé aux facteurs de la norme contrôle. Les résultats du groupe anorexique sont également très proches de ceux de la norme des patients présentant un TCA de la version anglaise de Garner. Le seul facteur s'éloignant de la moyenne établie par Garner est le facteur boulimie (valant 10,5). Nos participantes anorexiques obtiennent une moyenne inférieure à un écart-type $(4,75)$. Ce résultat n'est pas étonnant puisque la norme de la version anglaise inclut des patients souffrant de divers TCA (anorexie, boulimie et hyperphagie) alors que notre échantillon ne comprend que des patientes anorexiques.

De manière générale, nous pouvons conclure que les résultats au questionnaire EDI-2 de cette recherche sont cohérents avec ceux rapportés dans la littérature [27,28], qu'il s'agisse du groupe contrôle ou du groupe de participantes anorexiques.

\subsection{Les résultats du questionnaire IDEA}

Les différences observées au questionnaire IDEA entre les groupes contrôle et anorexique sont en faveur des hypothèses phénoménologiques concernant le corps-pour-autrui (perturbation de l'identité, de l'incarnation et de l'intersubjectivité). Les quatre facteurs de l'IDEA ainsi que le score total varient de manière significative en fonction des participantes présentant ou non un diagnostic d'anorexie mentale (voir Fig. 2).

Le Tableau 2 indique également que les différences obtenues entre les facteurs du questionnaire IDEA sont très significatives (avec une probabilité de dépassement fixée à 0,05 ). De plus, la similarité des moyennes aux quatre facteurs de l'IDEA du groupe anorexique montre que les patientes anorexiques rencontrent bien des difficultés au niveau global de l'intersubjectivité : évaluation à travers le regard des autres (facteur GEO :2,22), à travers la privation (facteur $S: 2,73$ ), par des mesures objectives comme le poids et les mensurations (facteur OM : 2,65) et ressentent un sentiment d'étrangeté vis-à-vis de leur corps (facteur EB : 2,44). Si nous comparons ces résultats avec ceux du groupe anorexique de l'étude de Stanghellini et al. [19], nous remarquons qu'ils sont tous supérieurs à ceux obtenus dans l'étude de Stanghellini et al. [19].

Concernant la population contrôle, notre échantillon obtient des scores relativement élevés aux facteurs GEO (se sentir soi-même à travers le regard et l'évaluation des autres) et OM (se sentir 
Tableau 2

Test U de Mann-Whitney comparant les moyennes aux facteurs du questionnaire IDEA des groupes contrôle et anorexique.

\begin{tabular}{llll}
\hline & $\mathrm{U}$ & Z ajusté & Valeur $p$ \\
\hline Facteur GEO & 4833 & $-5,1937$ & $0,000001^{*}$ \\
Facteur OM & 2578,5 & $-8,641$ & $0,000001^{*}$ \\
Facteur EB & 1645 & $-10,0845$ & $0,000001^{*}$ \\
Facteur S & 1780,5 & $-9,8736$ & $0,000001^{*}$ \\
Score total & 2099 & $-9,3536$ & $0,000001^{*}$ \\
\hline
\end{tabular}

Facteur GEO = se sentir soi-même à travers le regard et l'évaluation des autres ; Facteur OM = se sentir soi-même à travers des mesures objectives ; Facteur $\mathrm{EB}$ = se sentir étranger à son corps ; Facteur $\mathrm{S}$ = se sentir soi-même à travers la privation alimentaire ; Score total $=$ score moyen au questionnaire

* Les valeurs $p$ sont toutes significatives $(p<0,001)$.

\section{Tableau 3}

Tableau des coefficients des corrélations de Spearman entre les facteurs du questionnaire IDEA et les facteurs respectifs des questionnaires EDI-2 et du questionnaire complémentaire du groupe anorexique.

\begin{tabular}{llllll}
\hline & Facteur GEO & Facteur OM & Facteur EB & Facteur S & IDEA Score total \\
\hline EDI-2 Facteur RM & $0,43^{*}$ & $0,69^{*}$ & $0,48^{*}$ & $0,74^{*}$ & $0,68^{*}$ \\
EDI-2 Facteur B & $0,36^{*}$ & $0,42^{*}$ & $0,27^{*}$ & $0,31^{*}$ & $0,44^{*}$ \\
EDI-2 Facteur IC & $0,28^{*}$ & $0,46^{*}$ & $0,41^{*}$ & $0,67^{*}$ & $0,47^{*}$ \\
EDI-2 Facteur SI & $0,27^{*}$ & $0,42^{*}$ & $0,70^{*}$ & $0,59^{*}$ & $0,53^{*}$ \\
EDI-2 Facteur P & 0,24 & $0,35^{*}$ & 0,22 & $0,34^{*}$ & $0,31^{*}$ \\
EDI-2 Facteur MI & 0,12 & $0,48^{*}$ & $0,40^{*}$ & $0,44^{*}$ & $0,35^{*}$ \\
EDI-2 Facteur CI & $0,24^{*}$ & $0,50^{*}$ & $0,64^{*}$ & $0,57^{*}$ & $0,51^{*}$ \\
EDI-2 Facteur PM & $0,30^{*}$ & $0,39^{*}$ & $0,40^{*}$ & $0,35^{*}$ & $0,43^{*}$ \\
EDI-2 Facteur A & $0,38^{*}$ & $0,69^{*}$ & $0,60^{*}$ & $0,65^{*}$ & $0,64^{*}$ \\
EDI-2 Facteur CP & $0,27^{*}$ & $0,50^{*}$ & $0,57^{*}$ & $0,56^{*}$ & $0,51^{*}$ \\
EDI-2 Facteur IS & $0,35^{*}$ & $0,51^{*}$ & $0,57^{*}$ & $0,57^{*}$ & $0,55^{*}$ \\
17 Items Facteur R & $0,36^{*}$ & $0,70^{*}$ & $0,61^{*}$ & $0,76^{*}$ & $0,65^{*}$ \\
17 Items Facteur V & $0,59^{*}$ & $0,56^{*}$ & $0,51^{*}$ & $0,54^{*}$ & $0,66^{*}$ \\
17 Items Facteur A & 0,24 & $0,48^{*}$ & $0,36^{*}$ & $0,50^{*}$ & $0,42^{*}$
\end{tabular}

Facteur GEO = se sentir soi-même à travers le regard et l'évaluation des autres ; Facteur OM = se sentir soi-même à travers des mesures objectives ; Facteur $\mathrm{EB}=$ se sentir étranger à son corps ; Facteur $\mathrm{S}=$ se sentir soi-même à travers la privation alimentaire ; Facteur RM = recherche de la minceur ; Facteur B = boulimie ; Facteur SI=sentiment d'inefficacité ; Facteur IC = insatisfaction corporelle ; Facteur $\mathrm{P}=$ perfectionnisme ; Facteur $\mathrm{MI}=$ méfiance interpersonnelle ; Facteur $\mathrm{CI}=$ conscience intéroceptive ; Facteur $\mathrm{PM}=$ peur de la maturité ; Facteur $\mathrm{A}=$ ascétisme ; Facteur $\mathrm{CP}=$ contrôle des pulsions ; Facteur IS = insécurité sociale ; Facteur $\mathrm{R}=$ regard ; Facteur $\mathrm{V}=$ visage ; Facteur $\mathrm{A}=$ altruisme.

* Les corrélations marquées sont significatives $(p<0,05)$.

soi-même à travers des mesures objectives) comparés à ceux de l'échantillon contrôle de l'étude originale [19]. En effet, bien que les scores de notre groupe contrôle restent significativement inférieurs aux scores respectifs du groupe anorexique, ils doivent malgré tout attirer notre attention. Le facteur GEO de notre groupe contrôle est de 1,59 et celui issu de l'étude de Stanghellini et al. [19] vaut 0,53 . Nous constatons donc que notre échantillon contrôle se définit davantage à travers le regard des autres que celui de l'étude source. De même, le facteur OM de notre étude s'élève à 1,11 et celui de l'étude originale à 0,42 [19]. Nos participantes ont donc plus tendance à s'évaluer à travers des mesures objectives. Cependant, malgré ces scores observés pour le groupe contrôle, les différences entre nos groupes contrôle et anorexique demeurent nettement significatives, montrant que ces caractéristiques sont plus présentes chez les patientes anorexiques.

\subsection{Les corrélations entre les facteurs des questionnaires EDI-2 et IDEA}

Sur les 55 corrélations obtenues entre les facteurs du questionnaire EDI-2 et le questionnaire IDEA, 52 s'avèrent significatives ( $p<0,05$, voir Tableau 3 ). Par rapport aux 52 corrélations significatives, deux explications sont possibles pour interpréter ces résultats. Soit les corrélations positives signifient que les deux questionnaires évaluent un même concept ou deux concepts proches, soit qu'ils évaluent des concepts différents mais apparaissant conjointement. Dans notre recherche, les deux explications 
semblent pertinentes mais varient selon les facteurs étudiés. Certaines corrélations positives entre les facteurs de l'IDEA et de l'EDI-2 peuvent s'expliquer par la proximité des concepts évalués (ce qui correspond à la première explication) ; c'est le cas des corrélations entre le facteur S (se sentir soi-même à travers la privation alimentaire) et les facteurs recherche de minceur (corrélation $=0,74$ ), insatisfaction corporelle $(0,67)$ et ascétisme $(0,65)$ de l'EDI-2. De même, le facteur recherche de minceur est très lié au facteur OM (se sentir soi-même à travers des mesures objectives) avec une corrélation de 0,69 . Le facteur EB (se sentir étranger à son corps) corrèle avec les facteurs sentiment d'inefficacité $(0,70)$ et conscience intéroceptive $(0,64)$ de l'EDI- 2 . En d'autres termes, ce résultat suggère que plus le sentiment d'inefficacité est grand et moins la conscience de soi est bonne (correspondant à un score haut), plus la personne aura tendance à se sentir étrangère à elle-même. Par rapport au score total de l'IDEA, celui-ci est très associé aux facteurs ascétisme et recherche de la minceur, avec des corrélations respectives de 0,64 et 0,68 . Pour les autres corrélations, la deuxième explication semble davantage envisageable : il s'agit de concepts différents apparaissant de manière conjointe.

\section{Discussion}

Les résultats de notre étude démontrent une association globale entre les facteurs de l'IDEA et les facteurs de l'EDI-2. Celle-ci permet de suggérer que le questionnaire IDEA est bien en mesure d'identifier des phénomènes psychopathologiques liés à des traits typiques des patientes souffrant d'anorexie mentale.

Ce questionnaire permet d'identifier, en ayant recours à une perspective en première personne, les sujets présentant une psychopathologie anorexique avec le mérite de s'adresser aux patientes en évoquant des signes cliniques caractéristiques moins fréquemment évoqués mais pourtant centraux dans ce trouble. L'hypothèse de recherche (des résultats significativement supérieurs aux deux questionnaires pour le groupe de participantes souffrant d'anorexie mentale) est donc confirmée. En effet, toutes les différences observées entre les participantes contrôles et anorexiques sont significatives et plaident en faveur d'un vécu subjectif corporel et relationnel (le corps-pour-autrui) perturbé chez ces dernières.

Si l'on procède à une analyse plus spécifique des quatre facteurs du questionnaire, nos résultats permettent de proposer une sémiologie phénoménologique, en première personne, qui présente l'avantage de s'écarter de la préoccupation alimentaire (ce qui correspond généralement au discours des patientes). Les personnes anorexiques montrent ainsi qu'elles ont particulièrement besoin du regard des autres et de l'évaluation de ces derniers pour se sentir elles-mêmes (facteur 1 - GEO). Leur vécu corporel serait ambigu puisqu'il reposerait sur un rapport d'étrangeté à celui-ci (facteur 3 - EB), ce qui conduirait à pouvoir se sentir soi-même à travers des mesures objectives et non pas un ressenti en première personne véritable (facteur 2 - OM). Ce vécu corporel idiosyncrasique aurait pour conséquence que le sujet anorexique se sent davantage être lui-même à travers des actes de privation et de maîtrise (facteur 4 - S).

Notre étude suggère qu'autrui a une place particulière dans l'expérience anorexique. Comme nous le disait Jeanne, patiente anorexique : "si je vivais seule sur une île déserte, je n'aurais jamais souffert de ce trouble " [22]. Le regard qui est posé par autrui sur la personne anorexique repose sur un paradoxe puisque, si celle-ci est particulièrement dépendante de ce regard (facteur GEO), ce dernier peut être vécu comme réprobateur ou dévalorisant. C'est précisément ce que Sartre propose à travers le concept de corps-pour-autrui, en identifiant l'ambivalence de l'expérience du regard posé sur soi et de l'objectification qu'elle provoque [" Par le regard d'autrui, je me vis comme figé au milieu du monde » ([24], p. 307). L'anorexique serait particulièrement sensible au regard dévitalisant que l'autre lui porte tout en étant dépendante et attentive à celui-ci [23]. Les comportements altruistes (y compris d'altruisme alimentaires) classiquement associés au trouble $[10,11]$ peuvent également trouver leur source dans ce rapport ambivalent à autrui. Nous rejoignons également l'hypothèse d'un vécu de " perte de l'évidence corporelle " proposée par Wykretowicz et al. [23], démontrant que l'enjeu primordial de l'expérience anorexique est corporel et répond à un vécu incertain d'une perte d'aisance et de jouissance du corps en tant que base stable permettant de se sentir soi et d'interagir avec autrui. Ces propositions nous semblent rencontrer d'importantes pistes psychothérapeutiques qui auraient 
pour recommandation de prendre en charge le corps, au-delà de la dimension nutritive et biologique, en tant que mode d'expression et possibilité d'interaction.

Les perspectives qu'offre la phénoménologie permettent d'envisager des propositions de prise en charge et des modèles thérapeutiques faisant de la problématique du corps et du corps-pourautrui une dimension cardinale. Il est déterminant de comprendre qu'une approche thérapeutique centrée sur une approche phénoménologique se différencie d'une approche thérapeutique cognitivocomportementale traditionnelle (voir par exemple Fairburn, [29]) en ce qui concerne la place du corps et la manière de considérer ce dernier. Nous devons, en effet, garder à l'esprit que le discours sur le corps donne accès à la représentation que le sujet a de son corps, mais n'aborde la dimension corporelle vécue qu'indirectement. La perspective phénoménologique permet de ne pas réduire le corps à sa dimension strictement naturaliste (le Körper) ou encore aux représentations que le patient a de son corps. On peut d'ailleurs suggérer qu'une telle tendance est de nature à augmenter l'objectification du corporel au détriment de l'expérience vécue en première personne.

La prise en considération du " corps phénoménologique " (Leib et corps-pour-autrui) suggère de diminuer la focalisation sur le discours et la réflexivité à propos du corps biologique. Des modélisations thérapeutiques impliquant le corps et le corps du thérapeute doivent être envisagées afin de mettre en scène de façon directe l'expérience corporelle et son vécu, sans systématiquement passer par le discours, la représentation, voire la mentalisation. En outre, une diminution de l'attention sur le trouble alimentaire a également pour incidence de rencontrer le discours du patient et est, dès lors, susceptible d'améliorer le processus d'alliance thérapeutique souvent complexe à construire et entretenir avec ces patients.

\section{Conclusion}

Au vu de nos résultats, il semble que la traduction française du questionnaire IDEA [19] soit en mesure de discriminer une population anorexique d'une population sans TCA car les résultats des quatre facteurs diffèrent entre les groupes anorexiques et contrôle.

Cette recherche permet de confirmer les hypothèses phénoménologiques selon lesquelles les patientes anorexiques présentent un rapport à l'intersubjectivité entravé par le biais d'un déséquilibre au niveau du couple corps-objet et corps-sujet. En effet, les participantes anorexiques obtiennent des résultats significativement supérieurs à ceux des participantes sans TCA aux quatre facteurs du questionnaire IDEA mesurant différents aspects du concept du corps-pour-autrui. Ainsi, confrontées au regard des autres, les patientes interrogées se sentent dépourvues de leur subjectivité corporelle et, dans une certaine mesure, dépossédées de leur identité. Leur corps-pour-autrui prend donc une place démesurée et il semble raisonnable de dire que ce dernier est un concept central dans l'organisation psychopathologique de l'anorexie.

\section{Déclaration de liens d'intérêts}

Les auteurs déclarent ne pas avoir de liens d'intérêts.

\section{Annexe 1. Version francophone du questionnaire IDEA (IDentity and EAating disorder)}

\begin{tabular}{llllll}
\hline & Pas du tout & Légèrement & Moyennement & Beaucoup & Fortement \\
$(3)$ & $(0)$ & $(1)$ & $(2)$ & $(4)$ & $(3)$ \\
\hline
\end{tabular}

1. Pour moi, c'est très important de me voir à travers le regard des autres.

2. Manger selon mes propres règles est la seule manière de me sentir moi-même.

3. Si je ne peux pas manger "à ma façon", je ne me sens plus moi-même.

4. Parfois, je ressens des émotions qui me sont étrangères et qui m'effraient. 
5. Si mes mensurations ou mon poids restent stables dans le temps, je me sens moi-même, sinon, je me sens perdu(e).

6. J'ai une perception confuse de moi-même, comme si mon corps n'avait pas de limites nettes.

7. Parfois, j'arrive à définir qui je suis grâce au regard des autres.

8. Me voir selon le point de vue d'autrui me rend très nerveux/nerveuse.

9. Dans les situations de confusion, savoir que mon poids est sous contrôle me rassure quelque peu.

10. C'est seulement si mon poids est sous contrôle que j'apprécie d'être regardé(e) par autrui.

11. Mon bien-être et mon moral sont influencés par la manière dont je sens que les autres me regardent.

12. Mon corps en tant que chair n'est pas important.

13. Je suis dépendante de l'évaluation d'autrui.

14. Je n'ai pas une image claire et précise de moi-même, je ne me sens pas véritablement moi-même.

15. Savoir ce que les autres pensent de moi m'apaise, me rassure.

16. Si je pense que les autres n'ont pas une bonne opinion de moi, je préfère quand même toujours le savoir.

17. Si je suis les recommandations diététiques de mon programme de soins, je ne me reconnais pas quand je me regarde dans le miroir. Cela ne se passe pas ainsi lorsque je gère mon alimentation à ma façon.

18. Je ne supporte pas de ne pas savoir ce que les autres pensent de moi.

19. Changer mes habitudes alimentaires me terrifie comme n'importe quel autre changement dans ma vie.

20. La peur de changer est une émotion que je ne tolère pas.

21. Contrôler mon poids signifie avoir le contrôle sur d'éventuels changements de mon corps.

22. Contrôler mon poids me donne le sentiment de contrôler mes émotions.

23. Quand je rencontre quelqu'un, je ne peux pas m'empêcher de vouloir savoir ce qu'il pense de moi.

Facteur GEO = se sentir soi-même à travers le regard et l'évaluation des autres (items : 1, 7, 8, 11, 13, 15, 16, 18, 23); Facteur $\mathbf{O M}=$ se sentir soi-même à travers des mesures objectives (items : 5, 9, 10, 21, 22); Facteur $\mathbf{E B}=$ se sentir étranger à son corps (items : 4, 6, 12, 14, 20); Facteur $\mathbf{S}=$ se sentir soi-même à travers la privation alimentaire (items : 2, 3, 17, 19); Score total $=$ somme des scores aux quatre facteurs.

\section{Références}

[1] American Psychiatric Association. Diagnostic and Statistical Manual of Mental Disorders (Text, revision). 4th ed. Washington, DC: American Psychiatric Association Press; 2000.

[2] American Psychiatric Association. Diagnostic and Statistical Manual of Mental Disorders. 5th ed. Washington, DC: American Psychiatric Association Press; 2013.

[3] American Psychiatric Association. Highlights of Changes from, DSM-IV-TR to DSM-5. Washington, DC: American Psychiatric Association Press; 2013.

[4] Hoek HW, Van Hoeken D. Review of the prevalence and incidence of eating disorders. Int J Eating Disord 2003;34(4):383-96.

[5] Guelfi JD, Rouillon F. Manuel de psychiatrie. Paris: Elsevier Masson; 2012. 
[6] Jeammet P. Anorexie, boulimie : les paradoxes de l'adolescence. Paris: Hachette; 2004.

[7] Steinhausen HC. The outcome of anorexia nervosa in the 20th century. Am J Psychiatry 2002;159:1284-93.

[8] Gallagher S, Zahavi D. The phenomenological mind: an introduction to philosophy of mind and cognitive science. New York: Routledge; 2008.

[9] Englebert J. Psychopathologie de l'homme en situation. Paris: Hermann; 2013.

[10] Englebert J, Follet V, Valentiny C. Anorexia nervosa and first-person perspective: altruism, family system and body experience. Psychopathology 2018;51(1):24-30.

[11] Demaret A. Éthologie et psychiatrie. Bruxelles: Mardaga; 2014.

[12] Bowden H. A phenomenological study of anorexia nervosa. Philos Psychiatry Psychol 2012;19(3):227-41.

[13] Bowden H. "Too fat" and "too thin": understanding the bodily experience of anorexia nervosa. Philos Psychiatry Psychol 2012;19(3):251-3.

[14] Brogna P, Caroppo E. The body as a simulacrum of identity: the subjective experience in the eating disorders. Ann Ist Super Sanita 2010;46(4):427-35.

[15] Castellini G, Trisolini F, Ricca V. Psychopathology of eating disorders. J Psychopathol 2014;20:461-70.

[16] Legrand D. Object and others: diverting Heidegger to conceptualize anorexia. Philos Psychiatry Psychol 2012;19(3):243-6.

[17] Legrand D. M'être au monde : soi, objets et autres sujets dans l'anorexie mentale. Cercle Hermeneut 2012;18-19:161-6.

[18] Legrand D. A disease of doubling. Philos Psychiatry Psychol 2013;20(1):93-6.

[19] Stanghellini G, Castellini G, Brogna P, Faravelli C, Ricca V. Identity and Eating Disorders (IDEA): a questionnaire evaluating identity and embodiment in eating disorder patients. Psychopathology 2012;45:147-58.

[20] Stanghellini G, Trisolini F, Castellini G, Ambrosini A, Faravelli C, Ricca V. Is feeling extraneous from one’s body a core vulnerability feature in eating disorders? Psychopathology 2014;48(1):18-24.

[21] Gaete MI, Fuchs T. From body image to emotional bodily experience in eating disorders. J Phenomen Psychol 2016;47(1):17-40.

[22] Englebert J. Anorexie et intersubjectivité : étude phénoménologique et éthologique. Ann Medicopsychol 2015;173(8):659-64.

[23] Wykretowicz H, Saraga M, Stiefel F. À propos d'un trouble de l'évidence corporelle dans l'anorexie. Evol Psychiatr 2019;84(1):185-97.

[24] Sartre JP. L'être et le néant. Paris: Gallimard; 1943.

[25] Uher R, Rutter M. Classification of feeding and eating disorders: review of evidence and proposals for ICD-11. World Psychiatry 2012;11:80-92.

[26] World Health Organization. Body Mass Index classification; 2006. Available from: http://www.euro.who.int/en/ health-topics/disease-prevention/nutrition/a-healthy-lifestyle/body-mass-index-bmi.

[27] Garner DM. Eating Disorder Inventory 2 professional manual. Odessa: Psychological Assessment; 1991.

[28] Van Strien T, Ouwens M. Validation of the Dutch EDI-2 in one clinical and two nonclinical populations. Eur J Psychol Assess 2003;19(1):66-84.

[29] Fairburn CG. Cognitive Behavior Therapy and Eating Disorders. New York: Guilford Press; 2008. 